Penultimate version, please cite published version:

Forthcoming in Epistemic Dilemmas: New Arguments, New Angles.

K. McCain, S. Stapleford \& M. Steup (Eds) Routledge.

\title{
Epistemic Dilemma and Epistemic Conflict
}

\section{Introduction}

When philosophers think about epistemic dilemmas, they usually think about certain evidential situations in which epistemic principles appear to give incompatible recommendations, render all available options impermissible, or lead to irrational outcomes in some other way. The main question about epistemic dilemmas is whether there can be evidential circumstances that make it inevitable for agents to violate at least one epistemic principle in their doxastic response to a proposition. Discussions about epistemic dilemmas, thus, primarily target the possibility that epistemic principles fail to do what they are supposed to do: justify or recommend at least one doxastic response relative to a specific body of evidence. In the debate on epistemic dilemmas, case descriptions are central. Advocates for the possibility of epistemic dilemmas aim to identify uncontroversial cases in which it is inevitable for agents to adopt irrational attitudes; opponents of the possibility of epistemic dilemmas aim to show that the above-presented cases can be resolved and thus do not pose genuine dilemmas.

In this paper, I will examine the notion of an epistemic dilemma, its characterizations in the literature, and the different intuitions prompted by it. I will illustrate that the notion of an epistemic dilemma is expected to capture various phenomena that are not easily unified with one concept: while some aspects of these phenomena are more about the agent in a certain situation, other aspects seem to be more about the situation as such. As a consequence, incompatible intuitions emerge concerning the transparency of epistemic dilemmas as well as regarding the role that the so-called third doxastic stance plays in resolving cases of epistemic dilemma.

I suggest we will be in a better position to answer the questions about transparency and the role of a neutral stance if we distinguish between the mental state of agents who find themselves in an epistemic dilemma and the normative situation that gives rise to a dilemma. I will refer to the agent's mental state as epistemic conflict and will reserve the term epistemic dilemma for evidential situations in which epistemic principles either recommend incompatible doxastic responses or render all options impermissible. The concept of epistemic 
conflict not only captures the mental state of agents who find themselves in a genuine epistemic dilemma but also applies to agents who face difficult epistemic choices that they cannot resolve without substantial cognitive (and often pragmatic) effort. I will spell out the relation of epistemic dilemma and epistemic conflict with the help of ideal agent $\Sigma$ and nonideal agent $S$. It will be illuminating to uncover the factors that allow epistemic conflict and epistemic dilemma to come apart for these two agents.

In Section 1, I will capture the notion of an epistemic dilemma as it is used in the literature and identify two relevant aspects. In Section 2, I will formulate a realistic case that gives rise to a genuine epistemic dilemma. I will use this case in Section 3 to show that there are diverging intuitions concerning the transparency of epistemic dilemmas and the role of suspension. In the fourth section, I will explicate the concepts of epistemic dilemmas and epistemic conflicts and show that this distinction can accommodate the different intuitions mentioned before.

\section{$1 \quad$ Being Caught in a Dilemma}

To better understand epistemic dilemmas, it is helpful to look at moral dilemmas first. One of the most famous cases that is supposed to constitute a moral dilemma is Sophie's Choice, and, although it is not optimal, ${ }^{1}$ I will use it here because it is so well known: Sophie, the mother of two children, is forced to decide which of her two children is going to live, and which is going to die. As their mother, Sophie obviously has a moral obligation with respect to each of her two children to protect them. Yet, no matter which one of the two she chooses to protect, she will inevitably fail to meet her moral obligation with respect to the other child. This situation is said to be a moral dilemma because all of Sophie's options are such that she will ultimately fail to meet her moral obligations. Sophie is trapped in this situation, and there is no way out of it.

Rational dilemmas appear to be structurally similar to moral dilemmas: in both situations, agents are unable to answer certain matters in accordance with the relevant norms because all options are in some sense impermissible. An epistemic dilemma arises with regard to the truth of a proposition in a particular evidential context. In an epistemic dilemma, agents cannot but violate at least one epistemic principle when making up their mind regarding $p$ 's truth, given the evidence. Typically, epistemic dilemmas are said to arise due to the recommendations of at least two epistemic principles that either jointly render all options

\footnotetext{
${ }^{1}$ What makes it not optimal is the fact that there is a third-party involved. It seems possible, though, to translate Sophie's Choice into a setting involving only natural forces.
} 
impermissible or pull in opposite directions given a particular evidential context. ${ }^{2}$ Here are some characterizations of epistemic dilemmas in the literature:

Let us agree that it is a sufficient condition for a person, $\mathrm{S}$, to be in an epistemic dilemma with regard to a proposition, $\mathrm{P}$, if S's epistemic reasons argue that $\mathrm{S}$ should not believe $\mathrm{P}$, should not deny P, and should not withhold judgment on P. (Conee 1994: 475)

In an epistemic impasse, you shouldn't adopt any doxastic attitude toward P, even though you're condemned to adopt one of them. (Turri 2012: 359)

There is a proposition such that, if one has any opinions about it at all, one will have a rationally unacceptable set of propositional attitudes - or if one doesn't, one will end up being cognitively imperfect in some other manner. (Brouwer 2014: 4451)

This paper is about epistemic dilemmas, i.e., cases in which one is doomed to have a doxastic attitude that is rationally impermissible no matter what. (Leonard 2020: 573)

According to these characterizations of epistemic dilemmas, there are two aspects that play a decisive role: (i) the agent somehow is required to respond doxastically, but (ii) is unable to do so because the epistemic principles fail to provide rational instructions on how to respond in the current evidential context. There are at least two questions to ask here: First, what kind of failure on the part of the epistemic principles is there and how does this failure lead to the agent's inability to respond rationally? Second, it is essential to ask where the requirement to respond doxastically is coming from and why it is not an option for the agent to simply not respond doxastically. I will address these questions in the next two subsections.

\subsection{Failure and Inability}

What kind of failure is there on the part of epistemic rationality such that agents are incapable of responding in a rational way? It seems that the problem for agents who are in an epistemic dilemma is not that the epistemic principles are merely silent on what to do. The problem, rather, is that, in a manner of speaking, they are talking too much. The agents' inability to

\footnotetext{
2 Turri (2012) distinguished between "epistemic impasse" and "epistemic dilemma": an impasse is a situation in which an agent has to choose between options, all of which are impermissible, whereas a dilemma is a situation in which an agent is obliged to adopt incompatible options. I will treat epistemic impasse as a special case of epistemic dilemma.
} 
rationally make up their minds about the truth of the proposition in question is not a result of the absence of recommendations provided by the epistemic principles; rather, it seems to be the presence of such recommendations that incapacitate the agents' ability to make rational epistemic choices.

Depending on whether epistemic principles are spelt out as obligations or permissions, the failure of epistemic rationality as it is supposed to occur in epistemic dilemmas can be stated in two different ways: First, epistemic rationality may fail if two or more epistemic principles are in conflict such that it is not clear (neither in the particular case nor in general) which principle takes precedence over the other(s). Taken together, the principles obligate agents to pursue rationally incompatible courses of action at the same time. Following this obligation would lead to "a rationally unacceptable set of propositional attitudes" (Brouwer 2014: 4451), whereas ignoring one's epistemic obligations would obviously be irrational as well. In such situations, the agent ends up being doxastically irrational, no matter what, and is thus unable to respond in a rational way.

Second, the failure of epistemic rationality can be described as each principle prohibiting at least one course of action such that, taken together, all available options are rendered impermissible by the obtaining principles (Conee 1994; Turri 2012). Whichever doxastic attitude the agent chooses, it will be rationally impermissible. At first glance, the impermissibility of all doxastic options seems less problematic than the obligation to do incompatible things because an agent's omission to adopt a doxastic attitude seems to be a permissible option after all. However, we need to distinguish between 'course of action' and 'adopting a doxastic attitude'. A genuinely dilemmic situation in terms of impermissibility concerns all courses of action, not just those that consist in the formation of a doxastic attitude. Thus, to show that there are genuine epistemic dilemmas, case descriptions that rely on impermissibility have to make sure that an agent's omission to adopt any doxastic attitude counts as an impermissible doxastic reaction as well. ${ }^{3}$ Only then it would be intelligible to say that the epistemic principles fail to provide rational instructions by rendering every possible option impermissible and that agents are thereby incapacitated to respond rationally. It is important to see that an omission to form any doxastic attitude regarding a proposition is a doxastic response in the sense that an agent cognitively relates to that proposition and does

\footnotetext{
${ }^{3}$ It seems that Conee (1994), in the quote above, takes "withholding judgment" to be exactly this: neither adopting the attitude of belief nor that of disbelief regarding a certain proposition. But if we take withholding or suspending to be a doxastic attitude itself, and not just the joint absence of doxastic attitudes (as has been recently proposed by Rosenkranz (2007), Friedman (2013a, 2017) and others), then proponents of epistemic dilemmas need to make sure that the rational principles applying in dilemmic cases do not permit adopting none of the three doxastic attitudes (believing, disbelieving and suspending).
} 
not form an attitude on the basis of her evidence. The mere absence of any attitude regarding $p$, however, is not necessarily a doxastic response because the mere absence of doxastic attitudes regarding some proposition may be the result of the subject's never having considered the truth of this proposition. ${ }^{4}$ Finding a case-description like this is not an easy task for the proponent of epistemic dilemmas. In the next section, I focus on this aspect of epistemic dilemmas: why is not forming an attitude not an option in these cases? Why is the agent required to consider the evidence regarding $p$ in the first place? Why not just opt out?

\subsection{Requirement to Respond Doxastically}

If we grant that epistemic dilemmas occur in evidential contexts in which all possible doxastic options are rendered irrational by the epistemic principles or in which the epistemic principles recommend incompatible courses of action, then it is sensible to say that agents in epistemic dilemmas are incapable to respond rationally. Whatever they do, they will do something irrational. However, it not yet clear what component of an epistemic dilemma makes sure that the agent is required to respond doxastically in the first place. This requirement to respond is needed to guarantee that the agent is trapped or caught in a dilemma. This idea that there is no way out is expressed by stating that an agent in an epistemic dilemma is "condemned to adopt" (Turri 2012: 359) an impermissible doxastic attitude, is "doomed to have an impermissible attitude" (Leonard 2020: 577), is "doomed to fall short of some rational ideal" (Christensen, 2010: 212), or that the agent's "cognitive shortfall is unavoidable" (Brouwer 2014: 4451). But where is this pressure to respond to a given proposition or question coming from? What feature of a case description that is supposed to be an epistemic dilemma plays the crucial role to condemn the agent to adopt an attitude or to make a doxastic choice in the first place? ${ }^{5}$

When we look at moral cases like Sophie's Choice, it is easy to see why Sophie is condemned to make a choice between her two children. Sophie faces the threat that both of her children are going to die if she refuses to choose one of them over the other. Thus, she is strongly motivated to make that choice to prevent the loss of both of her children. Without this threat, Sophie could easily avoid being caught in such a moral dilemma by just refusing to think it through. It seems that, to be caught in any kind of dilemma that arises in the context of settling a question, whether it is moral or epistemic, there needs to be a strong motivation

\footnotetext{
${ }^{4}$ See Bergmann (2004), p. 421: "I shall call these three attitudes, i.e., believing, disbelieving and withholding, as I have described it here, 'the doxastic attitudes'. Together with the possibility of taking no doxastic attitude at all, this gives four ways of relating to a proposition".

${ }^{5}$ Note that there is a distinction between being required to make a choice among different attitudes and being required to adopt a particular attitude. I am concerned with the former.
} 
to answer this question; it is this motivation that ensures that the agent is bound to answer the question and does not opt out prematurely. Only if the agent is bound to make a choice about how to respond will the fact that all options are prohibited lead to the situation that the agent is actually doomed to respond irrationally.

As we can see, the lack of epistemic guidance alone does not yet bring it about that an agent is caught in an epistemic dilemma. Why would anyone struggle with being unable to answer a certain question if there is no need to answer the question in the first place? Therefore, any case that describes an agent as being trapped in an epistemic dilemma has to say something about the agent's motivation to answer this particular question. ${ }^{6}$ The kind of motivation that is relevant here can but does not have to be purely intellectual. As long as there is no state-directed motivation to adopt a certain doxastic attitude regarding a proposition $p$ (e.g., believing that $p$ ), there is no problem with being pragmatically motivated to find the answer to the question whether $p .^{7}$

\section{$2 \quad$ A Sample Case}

Let me introduce an example case of an epistemic dilemma that satisfies the following conditions: It is a realistic case that does not include magical features, over-idealized cognitive abilities, and self-destructive or self-undermining evidence. Furthermore, in this case, the agent is required to respond doxastically with respect to the question whether a certain proposition $p$ is true, and the evidential context is such that either any doxastic response regarding $p$ is rationally prohibited, or the agent is rationally obligated to have incompatible doxastic attitudes.

REV-TEST Sarah is a student and took an online test with 10 yes/no questions. She is under pressure to achieve a score of $100 \%$ and took the test very seriously. When submitting her answers, she is confident that she answered all the questions correctly. The system reports that all but one question was answered correctly but does not say which of her answers is incorrect. Surprisingly, Sarah is given a one-time opportunity to reconsider her original answers. She is advised to either resubmit her set of answers as it was, or to revise it within a short period of time, during which she will not have access to any further information. After having thoroughly reviewed each of her

\footnotetext{
${ }^{6}$ Indeed, most case descriptions are such that the pressure of time, at least implicitly, plays some role: exams, footraces, logic tests and so on have to be taken or finished at a certain point of time (Christensen 2010; Foley 1991; Odegard 1993); pilots need to get down before they run out of fuel (Christensen 2010; Lasonen-Aarnio 2014); and donations, tips, and bets have to be made (Christensen 2010; Odegard 1993; Priest 2002).

${ }^{7}$ See Jonathan Kvanvig (2003: 41) who argued that "[w]e do have an interest in the truth, both pragmatic and purely intellectual."
} 
original answers $A_{1}$ to $A_{10}$, Sarah is unable to identify the incorrect answer based on her current evidence. ${ }^{8}$

Let us suppose that Sarah looks at each of her answers and asks herself: should I revise this answer? Rationally, Sarah is not permitted to pick an arbitrary answer and disbelieve its correctness because the original evidence that led her to this particular answer in the first round is still undefeated; she further has statistical evidence that this answer is correct (she knows that she has answered 9 out of 10 questions correctly); and she has almost no evidence that it is false (only 1 answer out of 10 is false). This clearly speaks in favor of believing that this particular answer is correct. Yet, the just-described evidential situation for this particular answer is the same for all 10 answers. If, on this evidential basis, she sticks to believing that this particular answer is correct, then she is rationally obligated to do the same for every other answer as well. However, she is rationally not permitted to believe that all of her answers are correct because she knows from the report that one of them is false. The crucial point is that she also cannot opt out and do nothing because she already adopted beliefs about each answer's correctness in the first round, and now, given the report in the second round, she additionally disbelieves that all answers are correct. In the face of the report, it would epistemically irrational not to revise her beliefs of the first round and just keep them. (As I will point out later, on the practical side, walking away and not doing anything is not an option, because then she would not pass the test at all.

If she disbelieves the conjunction that all of her answers are correct, as she is obligated to do given the report, she has to disbelieve one answer's correctness. However, given the current evidence, and there is no prospect of gaining any further evidence, there is no way to determine which answer that should be. It would also be irrational for Sarah to pick an arbitrary answer and disbelieve its correctness or suspend about it because there is no evidence that suggests picking this particular answer instead of any other, not to mention the evidence there is to believe of each single answer that it is correct. That means that either Sarah has to form an attitude against her evidence or, if she does not and sticks to her original answers, she has to violate simple closure principles. Sarah is indeed trapped in a situation in which, given her evidence, any doxastic response regarding the correctness of her individual

\footnotetext{
${ }^{8}$ REV-TEST is inspired by the Preface Paradox and by my students complaining that they could not see which of the questions they answered incorrectly in an online test they were allowed to repeat as often as they wanted within a certain time. Littlejohn (forthcoming) offers a similar example, in which agents deliberate about their performance in an eye exam. The main difference between his example and mine is that my example has an actual second round that requires the agent to make up her mind and revise on the basis of previous evidence and additional evidence provided by the report. Sarah cannot walk away from her task because she is pragmatically motivated to uncover and correct her mistake.
} 
answers leads to an epistemically irrational outcome. REV-TEST satisfies the condition that, no matter what Sarah is doing (or omitting to do), it will be epistemically irrational.

But why is she caught in that situation? Why not just leave the situation and not respond doxastically at all? If it were not for Sarah's strong motivation to revise her set of answers, she would not remain in this situation for long. Her motivation to score $100 \%$ explains why she remains in this hopeless situation and does not simply opt out. Sarah's motivation may be grounded in strong ambition, pure intellectual curiosity, or practical reasons like meeting her parents' expectations or not losing her stipend. Whether the motivation is pragmatic or epistemic does not play a role here, as long as the motivation is object-directed (getting it right) and not state-directed (adopting a certain attitude irrespective of its adequacy (e.g., as in Foley's (1991) example)). ${ }^{9}$ Because of her motivation, Sarah is trapped in a situation she cannot resolve.

Sure enough, Sarah's evidential context gives rise to a practical problem as well: because she needs to excel in this test, she cannot just resubmit her answers, knowing that one of them is false. She cannot arbitrarily revise one answer because she does not know which answer is incorrect, and the chance that she will pick an actually correct answer for revision, and thereby lower her score, is just too high, whereas the chance that she will pick the one incorrect answer for revision, and thereby score 100, is rather low. She also cannot walk away from this task and refuse to resubmit because that would mean that she would not pass the test at all. Whatever she does, she will fall short of her own ideals.

Note that even if Sarah encounters a practical problem, her situation does not qualify as a dilemma of practical rationality. Speaking in terms of maximizing utility, for example, it is clearly rational for Sarah to resubmit the set of answers as it was because the chance of worsening her original score is $9 / 10$, whereas the chance of being lucky and improving her score is only $1 / 10$. In this situation, it would be irrational for Sarah to take the risk and arbitrarily change one of her answers. Sarah is in an epistemic dilemma, though: she is under epistemic pressure to revise her current doxastic state, but none of the possible revisions are epistemically permitted. She cannot omit revising and do nothing because it would be incoherent to remain in disbelief that all ten answers are correct and, at the same time, believe each particular answer $A_{1}$ to $A_{10}$ to be correct. Whatever Sarah will be doing to revise her doxastic state, it will be impermissible given her evidence.

\footnotetext{
${ }^{9}$ Foley (1991) offered a situation in which agents are strongly motivated not to adopt a certain belief that they have adequate evidence for because adopting the belief would destroy that evidence. This is not the case here. Sarah is motivated to find the truth because she needs to know and has no interest in adopting a particular attitude.
} 
Although Sarah is in an epistemic dilemma but not in a practical one, one feature of the case description keeps her struggling practically and epistemically: she is strongly motivated to score 100 and it is psychologically not easy to let go of that aim. This motivation that keeps her from resubmitting the set of answers as it was, I argue, is the very same feature that keeps her trapped in the epistemic dilemma: she upholds the aim of finding out which answer is incorrect even though she cannot succeed.

\section{$3 \quad$ Intuitions and Problems}

\subsection{Transparency}

Being trapped in a (rational) dilemma, so it seems, essentially requires a conflict between one's aim to answer the issue (rationally) and one's inability to achieve this aim. This, I suggest, is the crucial intuition behind the idea that agents in an epistemic dilemma are necessarily aware of the problem that the epistemic principles prohibit every possible doxastic response in the respective evidential context. In my example case REV-TEST, Sarah is well aware of her situation and struggles to do the right thing doxastically. She is strongly motivated to find out for each individual answer whether it is correct or not, but she is unable to achieve this aim given her fixed evidential context. What keeps her trapped in this situation is her motivation to answer the question in combination with her knowledge that any doxastic response would be irrational in this evidential context. How else could agents be caught in a situation and struggle with their inability to answer a question if they do not know there is a problem?

Having said that, it seems false to say that one and the same evidential context gives rise to a dilemma for one agent but not for another agent, depending on whether or not they are aware of the rational obligations that apply in this situation. Is it not true for both agents that they cannot but respond irrationally in this context? Why would an agent's awareness of the relevant principles of rationality change this fact? If there is no rational way to respond, then not knowing about the problem will not evoke a rational response. Thus, it seems that we can pin down the relevant factors for a situation that give rise to an epistemic dilemma without requiring that an agent who is in that situation be aware of all of these factors. If it is true that some evidential contexts are such that the epistemic principles fail to recommend a rational doxastic response, this seems to be an objective fact. If we agree that REV-TEST is a case of genuine epistemic dilemma, then this status should not be contested, even if Sarah would be prepared to resubmit the original answers without any struggle; and it should not be contested, even if Sarah would revise her original position and end up disbelieving answer $\mathrm{A}_{3}$ 
just because three is her unlucky number. It seems sensible to say that Sarah would still be in a situational epistemic dilemma even if she does not struggle due to her misapprehension of what is rational given the evidence.

As I have shown, there are two essential aspects of the notion of epistemic dilemma that pull in opposing directions. How can an approach to epistemic dilemmas do justice to the natural connotation of dilemma as experienced struggle, on the one hand, and also address the need for an objective description of the evidential setting, on the other hand? In Section 4, I suggest distinguishing between epistemic conflict and epistemic dilemma to achieve this goal. Before doing so, I will discuss the intuition that taking a neutral stance can be helpful for resolving alleged cases of epistemic dilemmas.

\subsection{The Third Doxastic Stance}

For many situations of conflicting evidence, the so-called third doxastic stance has proven helpful. If agents lack sufficient evidence for believing a proposition $p$ and also lack sufficient evidence for disbelieving $p$, then they should take a neutral stance regarding $p$. Similarly, if there is equally balanced evidence for believing and for disbelieving $p$, then agents should remain doxastically neutral on the issue. Remaining neutral can be spelt out in different ways - for example, in terms of holding back a final verdict on the matter, in terms of bracketing one's beliefs in the presence of undercutting defeaters and opposed higher-order evidence, or in terms of adopting a neutral attitude with regard to the proposition in question. The debate on doxastic neutrality in terms of an attitude has gained traction in recent years, prominently by contributions by Sven Rosenkranz (2007) and Jane Friedman (2013, 2017).

It is tempting to think that a neutral stance could provide a way to resolve cases of alleged epistemic dilemma. Indeed, the third doxastic option besides believing and disbelieving is what makes the doxastic realm so special. Surely this must provide some help in dealing with alleged cases of epistemic dilemma. The suggestion that a neutral stance may neutralize dilemmas can be advanced in at least two ways: First, one could argue, among all the irrational options agents have at their disposal in cases of alleged epistemic dilemmas, a neutral stance, even if not perfect, is still the best one can do rationally (Conee 1994). Alternatively, one could argue that some form of neutrality is universally permitted and qualifies as a general fallback option.

Let us then consider whether a neutral stance would be helpful for Sarah. As I pointed out in Section 2, because the evidence is identical for each individual answer, Sarah cannot rationally adopt diverging doxastic attitudes with regard to answers $A_{1}-A_{10}$. Thus, if she 
believes one answer to be correct given the statistical evidence, then she has to believe of every other answer that it is correct as well. However, given her knowledge that one of the 10 answers is indeed incorrect, she cannot believe that all answers are correct and hence has to violate closure. Thus, what would be the best strategy to revise in a way that is compatible with her knowledge that one answer is incorrect? Picking one answer and believing it incorrect at will against the evidence seems not only psychologically unlikely but clearly irrational. Picking one answer and suspending with regard to its correctness is not an improvement at all. In both cases, Sarah would be required to neglect the evidence that speaks for the answer's correctness. Moreover, if only one answer is picked to be suspended upon, this would lead to different attitudes regarding her answers, despite having identical statistical evidence for all of them. Suspending with regard to the conjunction is clearly irrational as well because Sarah knows that exactly one answer is incorrect and therefore has to disbelieve the conjunction.

But what about suspending in every individual case? If Sarah suspends with respect to each individual answer's correctness, this is at least compatible with disbelieving the conjunction. Furthermore, with this consistent response, Sarah would not be guilty of having different attitudes regarding individual answers, despite having identical statistical evidence for each one of them. Hence, suspending with respect to the truth of each single answer while disbelieving their conjunction seems to be in some sense "the best" response to the evidence at hand. Unfortunately, however, there is still the local problem that suspending regarding the correctness of each single answer requires a disregard of the evidence that speaks clearly for believing it to be correct.

It may well be that, for this case, it is true that suspending with regard to each individual answer's correctness is in some sense better than all the alternatives, but that alone does not make it epistemically rational. Maybe there is another way of accounting for the intuition that suspending is in some sense better without claiming that the epistemic dilemma (if there is one) is actually resolved. In the next section, I will explain what this might look like.

\section{$4 \quad$ Distinguishing Epistemic Conflict and Dilemma}

In order to accommodate the intuitions regarding transparency and the role of a neutral stance, I suggest drawing a distinction between the normative situation that none of the possible doxastic responses is justified in a particular evidential context, on the one hand, and the mental state of an agent who is trapped in this normative situation, on the other. The 
characterizations of epistemic dilemmas as displayed in the quotations in Section 2 blend these two aspects into one notion of an epistemic dilemma. The aim of doing justice to both aspects is natural because case descriptions of epistemic dilemmas typically address the normative situation as well as the agent's mental state in that situation without explicitly saying so. My aim in the following is to separate the two aspects conceptually and to show under what conditions they may come together or apart in certain cases.

I will refer to the mental state of an agent who is trapped in an epistemic dilemma as epistemic conflict and to the normative situation as epistemic dilemma.

\subsection{Epistemic Dilemmas}

I define epistemic dilemmas as follows:

\section{(ED) Epistemic Dilemma}

An epistemic dilemma is a situation in a given evidential context in which epistemic principles either recommend the adoption of rationally incompatible doxastic attitudes regarding a certain proposition $p$ or prohibit all available doxastic options regarding $p$.

According to ED, my REV-TEST example describes a situation that qualifies as an epistemic dilemma, but that being so has nothing to do with Sarah in particular, the protagonist in this case. It is not relevant whether Sarah is rational, informed about the problem, motivated to answer the question, or struggling with her inability to answer the question; her evidential context is such that none of the possible doxastic responses is permissible to revise her currently incoherent (and thus irrational) doxastic state. We can apply this definition of epistemic dilemma to a particular agent who is in that situation as follows:

\section{Being in an epistemic dilemma}

If an agent $A$ is in an epistemically dilemmic situation, then $A$ is unable to respond rationally to the question whether $p$ on the basis of her evidence.

Again, to be in this situation, an agent does not have to be aware that the norms fail to provide an epistemically rational recommendation. There is no need for an agent to be trapped in a situation to be in that situation. The conditional can be true for epistemic agents who have no idea that the epistemic principles fail to recommend an epistemically rational response in their 
current evidential context in the way ED describes. $A$ 's inability to respond rationally is independent of whether or not $A$ knows that this is so. Thus, to address the intuition of struggle and being trapped in a dilemma, we need another notion that is about the particular agent's mental state.

\subsection{Epistemic Conflict}

I suggest that an agent who is trapped in a problematic epistemic situation, for example in a situation of epistemic dilemma, is in a mental state of epistemic conflict. I will define the mental state of an epistemically conflicted agent through the help of two necessary conditions that are jointly sufficient:

\section{(EC) Epistemic conflict}

An agent $A$ is in the mental state of epistemic conflict with regard to the question whether a proposition $p$ is true given her current evidential context if and only if

(i) $A$ is strongly motivated to answer the question whether $p$ is true,

(ii) $A$ finds herself unable to answer the question whether $p$ is true on the basis of her current evidential context, and

(iii) $A$ does not expect her current evidential situation to improve.

Conditions (i) and (ii) constitute the agent's struggle: $A$ is strongly motivated to answer a question but finds herself unable to do so. This is a common situation, for sure, and usually a reason to engage in further inquiry. Thus, a conflict only arises if further (decisive) evidence is not expected by $A$, for example because $A$ believes that the full body of evidence has been assessed already or because time is running out. This is what condition (iii) is adding. ${ }^{10}$

Note that epistemic conflicts and epistemic dilemmas do not necessarily apply to the same cases. As I indicated above, the fact that an agent is in a situation of epistemic dilemma in the sense of ED does not necessarily lead to that agent being epistemically conflicted in the sense of EC. Furthermore, an agent who is in the mental state of epistemic conflict is not necessarily in a situation of epistemic dilemma. Among other things, this is so because agents can misapprehend their evidential situation in two different ways: (a) they do not understand that they are in a dilemmic situation and wrongly take themselves able to answer the question; (b) they do not understand that they are not in a dilemmic situation and wrongly find themselves unable to answer the question. Likewise, agents can also be mistaken in their

\footnotetext{
${ }^{10}$ Thanks to Igor Douven for making me clarify this point.
} 
expectations regarding the prospect of future evidence as addressed in condition (iii). So, how are the two concepts of epistemic dilemma and epistemic conflict connected? The relation between epistemic conflict and epistemic dilemma can be described with the help of the concept of an ideal agent.

\section{Relationship of EC and ED}

An ideal epistemic agent $\Sigma$ is in the mental state of epistemic conflict with respect to the truth of a proposition $p$, given a certain body of evidence $E$ only if $\Sigma$ is in a situation of epistemic dilemma regarding the truth of $p$ given $E$.

Since the failure of an ideal epistemic agent to answer the question whether $p$ cannot be explained by a failure of the ideal agent's cognitive capacities, it will be the epistemic principles that have failed to recommend a rational doxastic response given the evidence at hand. An ideal agent $\Sigma$ does not find itself unable to answer a question in an evidential context if this question can, in principle, be rationally answered in this evidential context. If we stipulate that ideal agents have a strong intellectual motivation to settle any question they are confronted with, the connection between epistemic dilemmas and epistemic conflicts can be established in the other direction as well: in this case, it would be true that $\Sigma$ is in the mental state of epistemic conflict if and only if $\Sigma$ is in a situation of epistemic dilemma.

In REV-TEST, nonideal but sufficiently rational Sarah is epistemically conflicted regarding the question of how to revise her set of answers in light of the report that one answer is incorrect. She is conflicted because she is in an epistemic dilemma, understands her evidential situation, and is strongly motivated to answer the question. Sarah's case not only describes an epistemically dilemmic situation, in which all possible doxastic responses are rationally impermissible, but also illustrates what it is like to be trapped in this dilemma: Sarah finds herself unable to answer a question that she is strongly motivated to answer in a situation where she does not expect further evidence to decide the issue. Her experienced struggle manifests in the mental state of epistemic conflict.

The proposed distinction between epistemic dilemmas and epistemic conflicts can explain why intuitions diverge regarding the transparency of epistemic dilemmas, as I described in Section 3.1. On the one hand, the notion of an epistemic dilemma, in the sense of ED, describes Sarah's normative situation independently of her motivation, insight and cognitive capacity; on the other hand, the notion of epistemic conflict, in the sense of EC, 
captures the struggle of which she is painfully aware. The suggested distinction of EC and ED can do justice to both intuitions.

What about the third doxastic stance? How could the distinction between EC and ED help here? To appreciate the relevance of this distinction to the role of the neutral stance regarding epistemic dilemmas, it is crucial to see that the mental state of epistemic conflict is not only reserved for agents such as Sarah who actually are in a situation of epistemic dilemma. Epistemic conflict is a broader notion and also covers agents who find themselves unable to settle doxastically for some other reason. Most importantly, EC also covers cases in which agents are not yet ready to adopt a neutral stance, even though the epistemic principles recommend or allow for doing so. I want to argue that, in some situations, it is cognitively and psychologically demanding for agents to take the rationally recommended or permissible neutral stance, especially if they have to do so against their strong motivation to answer the question. In some cases, the adoption of a neutral stance is psychologically not immediately available, even if it is normatively required or at least permitted. In contrast to ideal agents, nonideal agents may struggle with situations in which they are strongly motivated to answer a question, whereas the epistemic principles recommend neutrality. By contrast, in some cases, suspension is psychologically available even though it is normatively prohibited like in Sarah's case. Suspension, so I want to suggest, can thus be a way out of a conflict that is generated by a genuine dilemma. When agents are epistemically conflicted about a question and then suspend with regard to it, they deal with their epistemic conflict without resolving the normative situation that constitutes the dilemma. In the following, I will compare Sarah and Carl, who both deal with their epistemic conflict by suspending.

\subsection{Dealing with Epistemic Conflict}

In principle, there are two ways of resolving an epistemic conflict regarding the truth of a proposition $p$ : first, $A$ may believe or disbelieve $p$. Trivially, by having answered the question whether $p, A$ will not be motivated anymore to answer it. Moreover, by having answered the question, it is not true anymore that $A$ finds herself unable to answer it: condition (ii) is no longer met. Hence, the conflict disappears. Yet, unless the agent is able to exercise some sort of direct doxastic control to believe against the evidence, this is not really a promising method for resolving a conflict, especially when it is a conflict that is generated by a genuine epistemic dilemma.

Second, $A$ can resolve her conflict by giving up her aim to answer the question: condition (i) is no longer met. Psychologically, this can be done in different ways: $A$ may 
distract herself in ways that result in cognitive overload, confusion, or similar states. Sometimes this will work, and $A$ will forget or at least temporarily not think about the question. In such cases, $A$ is still unable to answer the question, but she does not struggle with her inability anymore (as long as the distraction works, in any case). Another more interesting way to give up one's aim of answering the question is by taking a neutral stance and settle one's indecision. ${ }^{11}$ By suspending, so I want to suggest, the question whether $p$ is being closed without having answered it. ${ }^{12}$ Again, $A$ will still be unable to answer the question whether $p$, but, due to overcoming her motivation to answer it, $A$ does not struggle with her inability anymore, but rather accepts that she is unable to answer the question. Yet, coming to terms with one's own inability and setting aside one's strong motivation to answer a question is cognitively effortful and can take some time.

In the following, I want to compare Sarah and Carl, who are both in a state of epistemic conflict, and deal with this conflict by suspending. Consider Carl's situation:

COV-TEST Carl works at a hospital and recently developed symptoms that could indicate an infection with COVID-19. The hospital and all the labs around are short of test-kits and are forced to stop testing medical staff until new material arrives in a fortnight. As it happens, Carl took the very last test available. To make things worse, his test was contaminated or lost such that he will never learn the result.

Clearly, Carl cannot answer the question whether he is infected or not, but he is strongly motivated to answer it. He needs to know soon to make important practical arrangements such as going back to work or isolating at home. Neither can he afford to spread the infection among his patients and colleagues, nor can he afford staying home with a simple cold when he is needed so badly. The epistemic norms clearly suggest taking a neutral stance on the question whether he is infected because there is insufficient evidence. An ideal agent (although unable to catch COVID-19) would do just this and act on the assumption that an infection cannot be ruled out. However, Carl is not an ideal agent and struggles with his inability to answer the question. He goes back and forth and tries to find some clues he might be missing that could answer the question and ease his mind. Only after some cognitive effort, Carl is psychologically ready to do the prudent thing: setting aside the nagging question and

\footnotetext{
${ }^{11}$ In 'Agnosticism as settled indecision' (Wagner forthcoming), I explain in detail what settling one's indecision is about. I argue that epistemic subjects commit to their de facto state of indecision by evaluating it as appropriate such that inquiry is closed. This account is open for suspending for pragmatic reasons as is needed in Sarah's case.

${ }^{12}$ There is an interesting discussion about different kinds of suspension some of which are inquiry-closing attitudes whereas others are inquiry-starting. See, for example, Staffel (2019). In Wagner (forthcoming), I explain why only inquiry-ending suspension can lead to the settled state of agnosticism.
} 
accept his own indecision. He then can take precautions and isolate on the basis of not being able to rule out an infection and the risks that come with it. Carl gives up his aim to answer the question (condition (i)) and thereby deals with his epistemic conflict. By suspending, Carl closes the question (at least for now) without answering it.

In contrast to Carl, Sarah is epistemically conflicted because she is caught in an epistemic dilemma. She knows that, given her evidential context, she is unable to answer the question that she is strongly motivated to answer: how to revise her set of answers in light of the report? Unlike Carl, Sarah is epistemically required not to suspend and not to have no attitudes regarding the correctness of her answers. However, as I said in Section 3.2, suspending with respect to each individual answer's correctness while disbelieving the conjunction seems somehow better than all the other options. This thought may be explained if we think of suspension as a good way to deal with epistemic conflict even though it is not a way to resolve an epistemic dilemma. It is still epistemically irrational to be neutral regarding the correctness of any single answer because there is good statistical evidence for each answer that it is true. However, it seems to be the best thing that Sarah can do to deal with her epistemic conflict because her struggle keeps her from resubmitting the set of answers as it was. By giving up her aim to get every question right, I want to claim, Sarah can focus on practical considerations such as maximizing utility: she can calculate the odds to maximize her score without being bothered by her aim of getting it right in every case. This is not to say that suspending is required to engage in such calculations; however, giving up the aim of getting it right $100 \%$ makes things easier for Sarah, such that she can resubmit the original set of answers despite knowing that one answer is false. It is thus psychologically plausible that it takes some effort to overcome this struggle. After having suspended (and thereby given up on her aim to score 100), Sarah remains in a situation of dilemma, but she is no longer caught in it.

Sarah and Carl are both in the mental state of epistemic conflict. Although Sarah's conflict is the consequence of her being caught in an epistemic dilemma, Carl ended up being epistemically conflicted due his own incapacity to follow the rational recommendation to suspend (or not form an attitude at least). Yet, for both agents, their respective motivation to answer the question hinders them from making practical decisions because these depend on their respective doxastic verdict. Carl has to decide whether to stay home or go to work, depending on his doxastic stance regarding his infection with COVID-19, and Sarah has to submit her answers with or without a revision, depending on her doxastic stance regarding each individual answer's correctness. In the end, Sarah and Carl deal with their epistemic 
conflict in the same way: by suspending. They both give up their aim to answer the question and thereby regain their ability to act regarding the practical problem. In suspending, Carl does the rationally permissible thing, epistemically as well as pragmatically speaking, whereas Sarah, by suspending, is not epistemically rational, but perhaps pragmatically rational.

This may explain the intuition that suspending is in some sense "better" than all the other options. Suspending is the best option in light of the practical problem that is a consequence of Sarah being caught in the epistemic dilemma. By dealing with her epistemic conflict, Sarah is in a better position to handle her practical problem; however, suspending is not a means to resolve the normative situation, that is, the epistemic dilemma itself. Instead of resolving an epistemic dilemma by suspending, Sarah merely frees herself from the dilemma's grip and stops being caught in it. While she cannot change the dilemmic normative situation, she can influence her state of mind with respect to this situation.

It is interesting to see that dealing with epistemic conflict in this way is not an option for ideal agents if the conflict is generated by a genuine dilemma; since this is the only way an ideal agent can be epistemically conflicted in the first place, ideal agents are never able to deal with their conflicts. Human irrationality seems to prove useful in situations in which rationality fails us.

\section{Acknowledgements}

I would like to thank Igor Douven, Thomas Müller, Daniela Schuster and Alexandra Zinke, as well as audiences in Konstanz and Tübingen for their feedback on earlier versions of this paper that helped improving it substantially. My special thanks go to the editors of this volume for inviting me to contribute to this volume. I want to thank Scott Stapleford and Kevin McCain for providing me with insightful comments and helpful questions about my first draft. I thank the Baden-Württemberg Stiftung for financially supporting this research project. 


\section{References}

Bergman, Michael (2005). Defeaters and higher-level requirements. The Philosophical Quarterly, 55(220), 419-436.

Brouwer, T. N. P. A. (2014). A paradox of rejection. Synthese, 191(18), 4, 451-4,464.

Christensen, D. (2010). Higher order evidence. Philosophy and Phenomenological Research, $81(1), 185-215$.

Conee, E. (1994). Against an epistemic dilemma. Australasian Journal of Philosophy, 72(4), 475-481.

Foley, R. (1991). Evidence and reasons for belief. Analysis, 51(2), 98-102.

Friedman, J. (2013). Suspended judgment. Philosophical Studies, 162(2), 165-181.

Friedman, J. (2017). Why suspend judging? Noûs, 51(2), 302-326.

Kvanvig, J. (2003). The value of knowledge and the pursuit of understanding. Cambridge University Press.

Lasonen-Aarnio, M. (2014). Higher order evidence and the limits of defeat. Philosophy and Phenomenological Research, 88(2), 314-345.

Leonard, N. (2020). Epistemic dilemmas and rational indeterminacy. Philosophical Studies, 177(3), 573-596.

Littlejohn, C. (forthcoming). Dividing away doxastic dilemmas. In Nick Hughes (Ed.), Epistemic dilemmas (pp. \#\#). Oxford University Press.

Odegard, D. (1993). Resolving epistemic dilemmas. Australasian Journal of Philosophy, $71(2), 161-168$.

Priest, G. (2002). Rational dilemmas. Analysis 62(1), 11-16.

Rosenkranz, S. (2007). Agnosticism as a third stance. Mind, 116(461), 55-104.

Staffel, J. (2019). Credences and suspended judgments as transitional attitudes. Philosophical Issues , 29(1), 281-294.

Turri, J. (2012). A puzzle about withholding. Philosophical Quarterly, 62(247), 355-364.

Wagner, V. (forthcoming). Agnosticism as settled indecision. Philosophical Studies. 